\title{
SLOW EXTRACTION OF ELECTRON BEAM WITH COMBINATION OF THE THIRD ORDER RESONANCE AND RFKO
}

\author{
A. Noda, T. Sugimura, T. Shirai, Y. Iwashita, A. Morita, H. Fujita and H. Tongu \\ Institute for Chemical Research, Kyoto University, Uji, Kyoto 611-0011, Japan
}

\begin{abstract}
Stretcher mode of KSR has been experimentally tested. Up to now, beam spill longer than 8 second has been attained by reducing RF knockout power. Improvement of extraction efficiency will be realized by ramping of betatron tune and formation of closed-orbit-distortion without the effect of non-linear sextupole magnets to make aperture minimum at the first septum for extraction.
\end{abstract}

\section{INTRODUCTION}

From the point of view of utilizing for counter experiments, it is very important to stretch out the beam spill of the output from the linac with small duty factor. The maximum pulse width and repetition rate of the 100 $\mathrm{MeV}$ disc-loaded type electron linac at ICR, Kyoto University are $1 \mu \mathrm{sec}$ and $20 \mathrm{~Hz}$, respectively and the beam duty factor is $2 \times 10^{-5}$ at maximum. In this condition, output signal from the detector easily get overlapped and correct information cannot be obtained. In order to enlarge the duty factor, stretcher mode of KSR is proposed[1] and its slow beam extraction system utilizing the third order resonance together with RF knockout has been constructed as shown in Fig. 1. KSR stretcher mode has already been studied experimentally in these few years resulting a good duty factor above $90 \%[2,3]$. Recent experimental studies, however, revealed the fact that the beam enlarged in its betatron oscillation

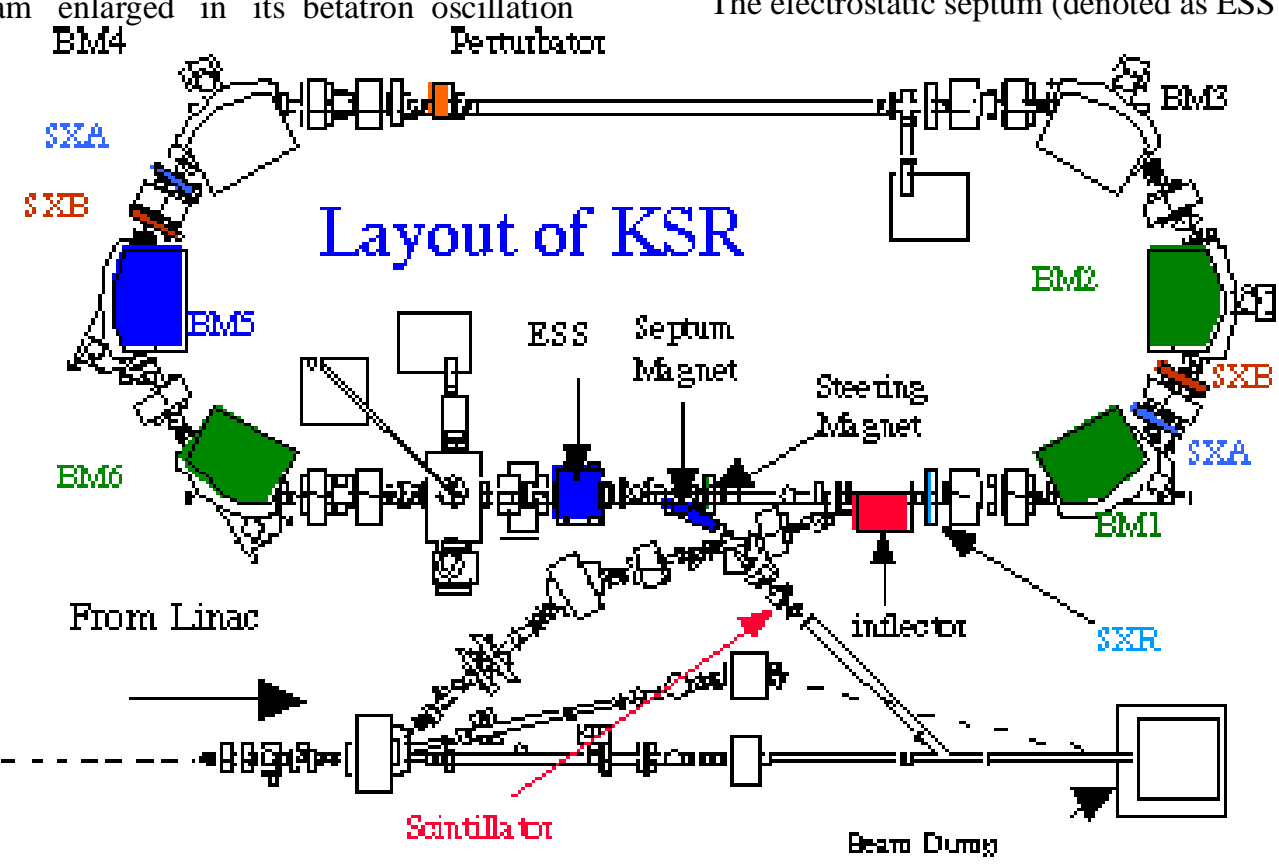

Fig. 1: Layout of KSR. Equipments for beam injection and extraction are emphasized by different colours. amplitude hits not only the first septum but also the septum of the inflector for beam injection, which should be avoided to attain good extraction efficiency. In the present paper, results of recent experimental investigation are presented together with the possible future improvement to realize good extraction efficiency.

\section{STRETCHER MODE OF KSR}

\subsection{Beam Injection}

The output beam of the $100 \mathrm{MeV}$ linac is injected into the KSR ring by making closed orbit distortion with use of a perturbator keeping the horizontal betatron tune a little bit apart from the third order resonance $2 \frac{1}{3}$ in order to form a separatrix as shown in Fig. 2 [1].

\subsection{Beam Extraction}

Injected beam into the separatrix (boundary between stable and unstable regions in the transverse phase space) is increased in its betatron oscillation amplitude by applying an external transverse RF electric field resonating with the betatron oscillation (called RF knock out hereafter) and is expected to reach the boundary of the separatrix and then get unstable and come out along the outgoing separatrices as shown in Fig. 2.

The electrostatic septum (denoted as ESS hereafter) is 


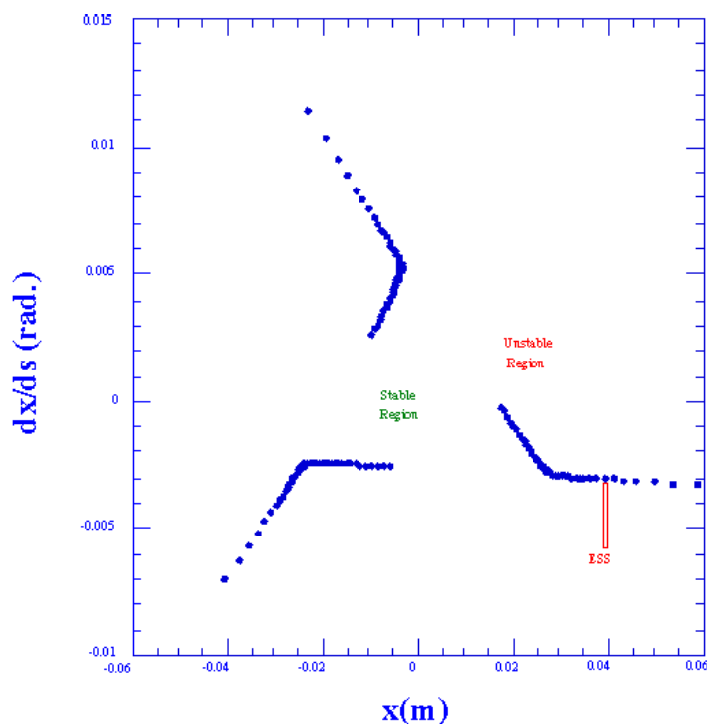

Fig.2 Phase space plot of the extracted beam

located $40 \mathrm{~mm}$ outward from the center of the ring aperture. The beam entered into the gap between electrodes of the ESS is designed to be deflected as large as $20.5 \mathrm{mrad}$ and is guided to the septum magnet located $\sim 1.5 \mathrm{~m}$ downstream as shown in Fig. 1, and then deflected by $45^{\circ}$ and is taken out from the ring. In Table 1 , main parameters of the slow beam extraction system are listed up.

\subsection{Closed Orbit Control System}

In order to attain good extraction efficiency, the entrance of the first septum (ESS) is required to make aperture minimum. As the extraction system locates at the same straight section as the inflector for beam injection, we applied an orbit distortion system with use of correction coils in dipole magnets, BM6, BM1 and BM2 together with the steering magnet (see Fig. 1) so as to assure the entrance of the ESS to be aperture minimum even in such a configuration,

If we denote the deflection angles by the correction coils in BM6, BM1, BM2 and the steering magnet as $\psi_{1}$,

Table 1 Main Paremeters of the beam extraction system

\begin{tabular}{|l|r|}
\hline \hline Electrostatic Septum(ESS) \\
\hline Deflection Angle & $20.5 \mathrm{mrad}$ \\
\hline Gap Electric Field & $70 \mathrm{kV} / \mathrm{cm}$ \\
\hline Electrode Length & $0.3 \mathrm{~m}$ \\
\hline Septum Thickness & $0.1 \mathrm{~mm}$ \\
\hline Gap Width \\
\hline Septum Magnet & $8 \sim 10 \mathrm{~mm}$ \\
\hline \hline Deflection Angle & $45^{\circ}$ \\
\hline Aperture (horizontal) & $29 \mathrm{~mm}$ \\
\hline Magnetic Field & $5 \mathrm{~T}$ \\
\hline Septum Thickness & $22.6 \mathrm{~mm}$ \\
\hline Pole Length & $550 \mathrm{~mm}$ \\
\hline Maximum Current & $1460 \mathrm{~A}$ \\
\hline Number of Coil Turns & 8 \\
\hline
\end{tabular}

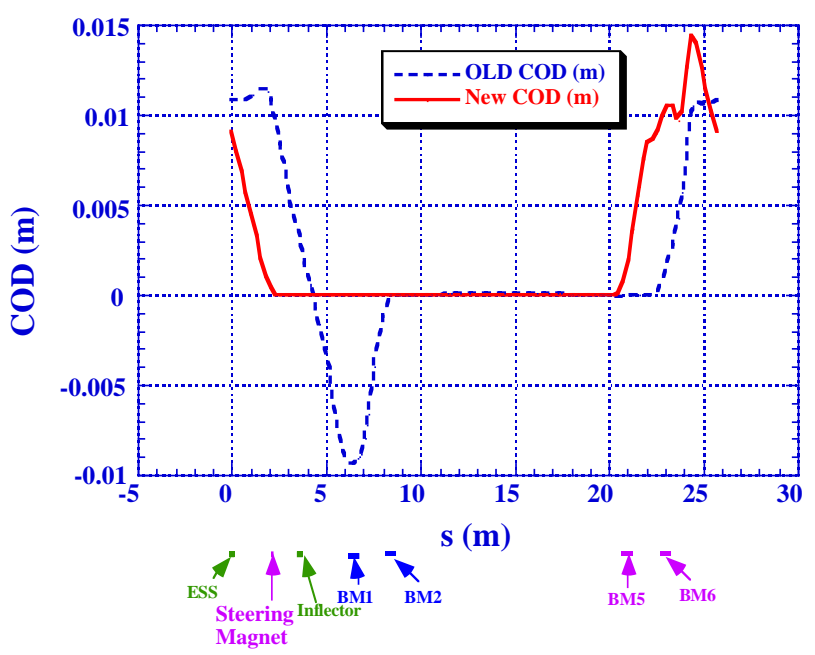

Fig. 3 Closed Orbit Distortion during and after injection. (calculation).

$\psi_{3}, \psi_{4}$, and $\psi_{2}$ respectively, then the required condition under linear optics is given by

$\sum_{i=1}^{4} \sqrt{\beta\left(s_{i}\right)} \cos \left\{v \pi-\left|\mu(s)-\mu\left(s_{i}\right)\right|\right\} \psi_{i}=0,\left(s \notin\left[s_{1}, s_{4}\right]\right),(1)$

where $\beta\left(\mathrm{s}_{\mathrm{i}}\right)$ and $\mu\left(\mathrm{s}_{\mathrm{i}}\right)$ are beta-function and betatron phase at the position $\mathrm{s}_{\mathrm{i}}$. (notation of $\mathrm{i}$ is the same as $\psi_{1}$ ). In Fig. 3, the closed orbit distortion when the excitation of the perturbator ended after injection is shown by a dashed line. From the linear calculation, the entrance of the ESS is expected to make aperture minimum.

\section{EXPERIMENTAL MEASUREMENT}

\subsection{Time Structure of the Extracted Beam}

The time structure of the extracted beam has been measured by a plastic scintillation counter set just downstream of the septum magnet. Its output signal is time structure analysed by a multi-channel scaler after discrimination [2]. In Fig. 4, typical examples of the measurements are given for the conditions with RF power of $0.09 \mathrm{~W}$ and $0.55 \mathrm{~W}$ for RF knockout. It is known that the beam spill time can well enlarged by reducing the RF power for RF knockout.

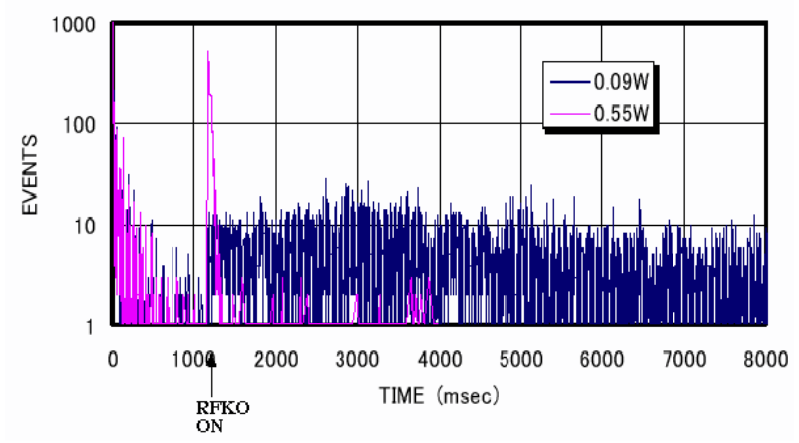

Fig.4. Time structure of the extracted electron beam 


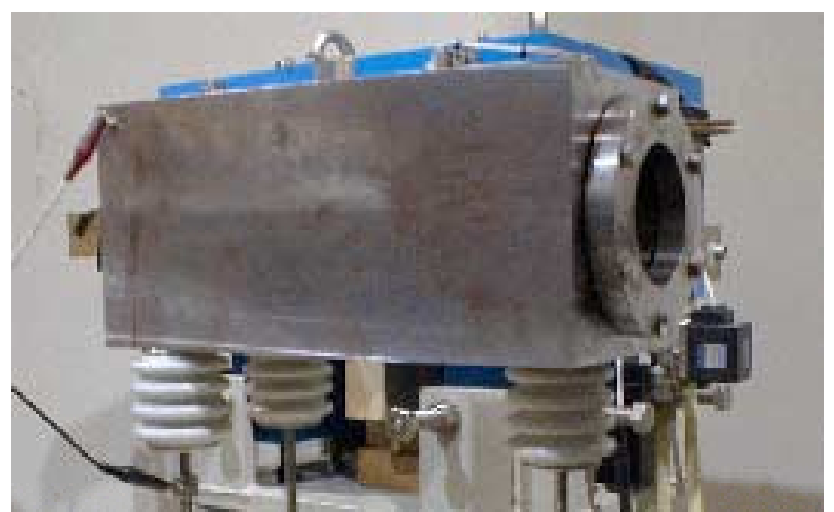

Fig. 5 Overall view of the fabricated Faraday cup.

\subsection{Extraction Efficiency}

For the purpose of evaluating the extraction efficiency, a Faraday Cup made of iron containing water inside in order to reduce the effect of outgoing shower was fabricated. In Fig. 5, an overall view of the Faraday cup is shown. It is calibrated with DCCT utilizing the output beam from the linac beforehand.

From the quantitative measurement, the extraction efficiency was found to be low and we investigated the reason. In Fig. 6(a), the time structure of the extracted electron beam observed at the extraction channel is shown. The similar observation when the scintillation counter is set at a position close to the inflector septum is shown in Fig. 6(b). The scale of vertical axis is the same in both Fig. 6(a) and (b), which suggests the situation the ESS does not make aperture minimum.

In order to inject the beam with some intensity, the separatrix size is required to be larger compared with the
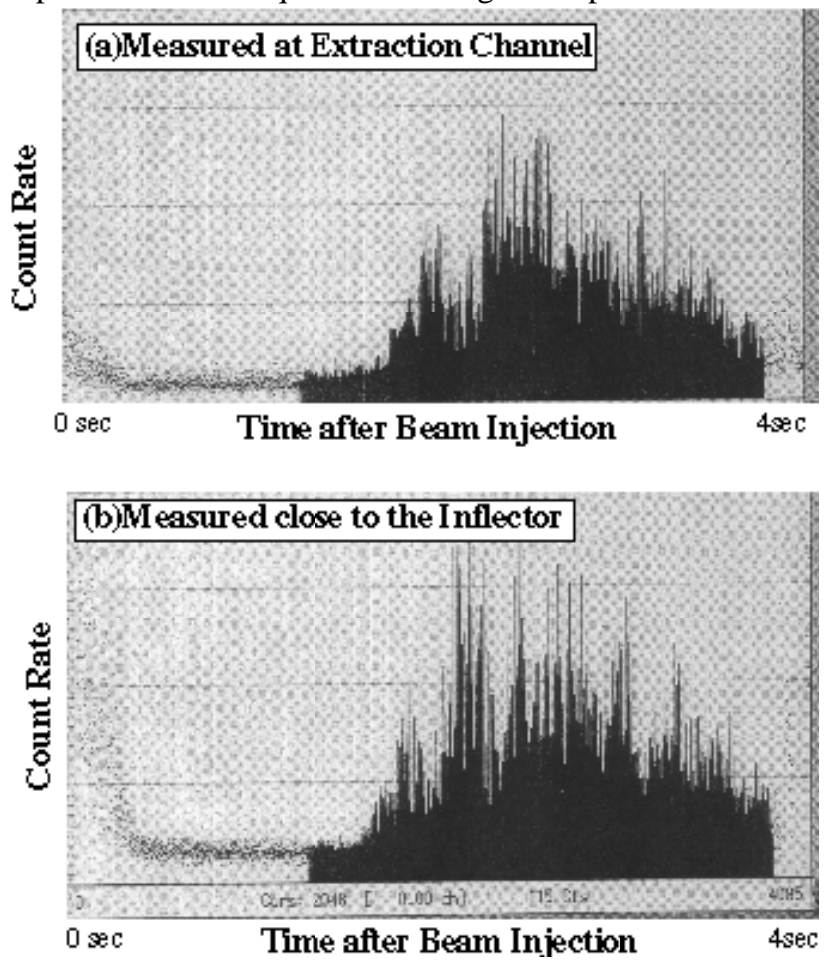

Fig. 6 Time structure of the extracted beam(a) and the one of electrons hitting the inflector (b).

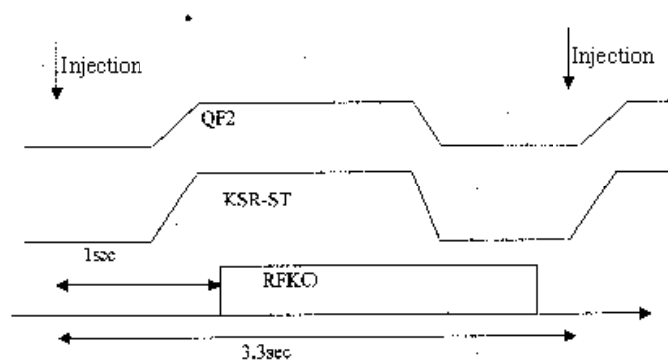

Fig. 7 Timing Chart for Ramping the betatron tune.

distance from the orbit center to the septum of ESS, which is different from the situation shown in Fig. 2. We consider that the septum locares in side the triangular region and electrons with different betatron oscillation phases can hit both the ESS and the inflector.

\section{POSSIBLE IMPROVEMENT}

\subsection{Ramping of Betatron Tune}

During beam extraction after RF knockout is switched on, it is required to set the septum position at the outgoing separatrix as shown in Fig. 2. For this purpose we push the horizontal betatron tune to the resonance $2 \frac{1}{3}$ so as to reduce the separatrix size, while the tune is kept a little bit apart from it during beam injection. We will apply ramping as shown in Fig. 7.

\subsection{COD Unperturbed by Sextupole Magnets}

Closed-orbit-distortion to make the aperture minimum at the entrance of ESS is found, however, to be much deformed due to presence of nonlinear sextupole magnets, SXR, SXA and SXB in the orbit distorted region. So as to avoid the nonlinear field effect, a new closed-orbitdistortion system is proposed. It is produced only in the region between BM5 and the steering magnet, where no nonlinear sextupole magnets exist. If we denote the deflection angle by the correction coils in BM5 as $\psi_{0}$, then the required condition is given by replacing domain of $i$ in (1) with 0 to 2 . In Fig.3, new closed-orbitdistortion is shown by a solid line.

Beam test with ramping of tune and a new closed orbit distortion system is scheduled in coming summar.

\section{REFERENCES}

[1] A. Noda et al., "Electron Storage and Stretcher Ring, KSR", Proc. of EPAC'96, Sitges (Barcelona), (1996) pp451-453..

[2] T. Sugimura et al., "Stretcher Mode Operation of KSR", Proc. of EPAC 2000, Vienna, Austria (2000)pp1002-1004.

[3] A. Noda et al., "Stretcher Operation of the $100 \mathrm{MeV}$ Disc-Load Electron Linac at ICR, Kyoto University", Proc. of the LINAC 2000, Monterey, California, USA (2000)pp624-62. 